\title{
Comparison of two surrogate estimates of insulin resistance to predict cardiovascular disease in apparently healthy individuals
}

\author{
M.R. Salazar ${ }^{\text {a,b,* }}$, H.A. Carbajal ${ }^{\text {b }}$, W.G. Espeche ${ }^{\text {a,b }}$, M. Aizpurúa ${ }^{\text {c }}$, C.A. Dulbecco ${ }^{\text {a,b }}$, \\ G.M. Reaven ${ }^{\mathrm{d}}$
}

${ }^{a}$ Hospital Universitario General San Martín, La Plata, Buenos Aires, Argentina

${ }^{b}$ Facultad de Ciencias Médicas, UNLP, Argentina

${ }^{c}$ Hospital Municipal de Rauch, Buenos Aires, Argentina

${ }^{d}$ Stanford University School of Medicine, Stanford, CA, USA

Received 17 July 2016; received in revised form 5 December 2016; accepted 12 December 2016

Available online 24 December 2016

\section{KEYWORDS \\ Insulin resistance; \\ Cardiovascular \\ disease; \\ TG/HDL-C ratio; \\ $\mathrm{TG} \times \mathrm{G}$ index; \\ Metabolic syndrome}

\begin{abstract}
Background and aims: Insulin resistance is associated with a cluster of abnormalities that increase cardiovascular disease (CVD). Several indices have been proposed to identify individuals who are insulin resistant, and thereby at increased CVD risk. The aim of this study was to compare the abilities of 3 indices to accomplish that goal: 1 ) plasma triglyceride $\times$ glucose index $(\mathrm{TG} \times \mathrm{G})$; 2) plasma triglyceride/high-density lipoprotein cholesterol ratio (TG/HDL-C); and 3) Metabolic Syndrome (MetS).

Methods and results: In a population sample of 723 individuals (486 women and 237 men, $50 \pm 16$ and $51 \pm 16$ years old, respectively), baseline demographic and metabolic variables known to increase CVD risk and incident CVD were compared among individuals defined as high vs. low risk by: TG $\times$ G; TG/HDL-C; or MetS. CVD risk profiles appeared comparable in high risk subjects, irrespective of criteria. Crude incidence of CVD events was increased in high risk subjects: 12.2 vs. $5.3 \%$ subjects/10 years, $\mathrm{p}=0.005$ defined by TG/HDL-C; 13.4 vs. $5.3 \%$ subjects/10 years, $\mathrm{p}=0.002$ defined by TG $\times \mathrm{G}$; and $13.4 \%$ vs. $4.5 \%$ of subjects $/ 10$ years, $\mathrm{p}<0.001$ in subjects with the MetS. The area under the ROC curves to predict CVD were similar, 0.66 vs. 0.67 for TG/ HDL-C and TG $\times$ G, respectively. However, when adjusted by age, sex and multiple covariates, hazard ratios for incident CVD were significantly increased in high risk patients classified by either TG/HDL-C ratio $(2.18, \mathrm{p}=0.021)$ or MetS $(1.93, \mathrm{p}=0.037)$, but not by $\mathrm{TG} \times \mathrm{G}$ index (1.72, $\mathrm{p}=0.087)$.

Conclusion: Although the 3 indices identify CVD risk comparably, the $\mathrm{TG} \times \mathrm{G}$ index seems somewhat less effective at predicting CVD.

(C) 2016 The Italian Society of Diabetology, the Italian Society for the Study of Atherosclerosis, the Italian Society of Human Nutrition, and the Department of Clinical Medicine and Surgery, Federico II University. Published by Elsevier B.V. All rights reserved.
\end{abstract}

\section{Introduction}

Insulin-mediated glucose disposal varies more than sixfold in volunteers with a normal medical history, physical examination, routine clinical laboratory tests, as well as a

\footnotetext{
* Corresponding author. 14 n 320, La Plata 1900, Buenos Aires, Argentina. Fax: +54 2214129164.

E-mail address: salazarlandea@gmail.com (M.R. Salazar).
}

normal oral glucose tolerance test [1]. Approximately onequarter to one-third of the most insulin resistant segment of such a population is at significantly greater risk [2-5] of a number of metabolic abnormalities known to increase risk of cardiovascular disease (CVD). Although it seems clinically beneficial to identify these high risk individuals in order to attempt interventions that might prevent manifest disease, how best to accomplish this goal is not self-evident. 
The plasma insulin concentration in response to a $75 \mathrm{~g}$ oral glucose challenge in apparently normal individuals is the surrogate estimate of insulin resistance most closely correlated to direct measures of insulin resistance $[1,6]$. However, the absence of a standardized clinical insulin assay prevents establishment of a specific numerical cutpoint that can be of general use to identify insulin resistant individuals [7]. Another approach is to use standardized measurements of changes closely associated with insulin resistance, and the characteristic dyslipidemia of insulin resistant individuals, high triglyceride (TG) and low high-density lipoprotein cholesterol (HDL-C) concentration, provided an attractive alternative [8]. The plasma concentration ratio of TG/HDL-C is significantly correlated with a direct measure of insulin-mediated glucose disposal [6], and sex-specific cut-points have been shown to identify apparently healthy individuals (without diabetes or CVD event) with cardio-metabolic risk profiles comparable to what was seen when these individuals met the diagnostic criteria for the metabolic syndrome (MetS) [9-11].

Guerrero Romero et al. [12] have taken a somewhat similar approach, using the fasting plasma TG and glucose concentration to create a mathematical formula (TG $\times \mathrm{G}$ index) to serve as a surrogate estimate of insulin resistance, and shown that this value is significantly correlated with euglycemic clamp measurements of insulin action. More recently, data have been published showing that the TG/HDL-C ratio and the TG $\times$ G index are significantly correlated to a similar degree with insulin-mediated glucose disposal using the insulin suppression test [13].

Both of these approaches were initially evaluated because of their relationship to insulin resistance, but their ultimate clinical utility will depend upon their ability to serve as simple clinical measures with which to identify individuals at increased risk of diabetes, hypertension, and/or incident CVD associated with insulin resistance [2-5]. In that context, the TG/HDL-C ratio has previously been shown to predict incident CVD in apparently healthy persons [11]. More recently, Sánchez-Iñigo et al., have shown that the TG $\times$ G index can predict incident ischemic heart and peripheral artery disease [14]. Both approaches seem comparable in terms of their simplicity to identify a subset of individuals at significantly enhanced risk of developing CVD. However, the fact that they are similarly associated with a direct measure of insulin resistance [13] does not mean that they would be equally effective in predicting incident CVD. Thus, the current analysis was initiated to address this issue by comparing the ability of the TG $\times$ G index, the TG/HDL-C ratio and MetS criteria [15] to predict CVD outcome in individuals without diabetes or previous CVD event.

\section{Methods}

\section{Study population}

In 2003, as part of a community intervention program focused on cardio-metabolic risk factors, an epidemiological study was conducted in Rauch city, Buenos Aires,
Argentina (RAUCH project, phase 2). According to the last national census available at the time of the survey, there were 8246 individuals $\geq 15$ years old in the urban area of Rauch. The city lies in a rural area in the Centre-Southeast region of the province of Buenos Aires and the population is primarily of European origin. Blocks of the urban area of Rauch city were randomly selected; because there were no differences in socioeconomic factors or the number of inhabitants within the city, a proportional probability was not taken into consideration. Previously trained, nurses from Hospital Municipal of Rauch conducted the survey in the subject's place of residence. They went to the selected homes (on up to 3 occasions when necessary to measure BP, weight, and height and construct an epidemiological chart. To minimize the chance of rejection, before the study a diffusion campaign was performed that delivered written information about the study to each selected residence. Because the design was a prospective cohort study, the sample size was determined taking into account the possibility of losing track of subjects during the study. Twenty percent of the inhabitants were regarded as a suitable and affordable sample according to the economic resources available. The survey was performed from subjects between 15 and 80 years old who lived in chosen blocks $(n=1308$, 855 women $51 \pm 17$ years old and 453 men $52 \pm 16$ years old, P between sexes $=0.63$ ). Fifty-four subjects who had previously suffered CVD events were excluded. All measurements necessary for the present study were available in 926 individuals (622 women and 304 men).

In 2012, 796 individuals (86\% of the baseline sample), 527 women and 269 men (or their relatives in case of death), could be surveyed again to obtain information concerning incident CVD events; the remaining inhabitants $(n=130)$ could not be found because they had moved from Rauch city. As previously published [11], there was no significant difference in the baseline characteristics between subjects with and without follow-up period. In order to avoid the effects of outliers, individuals with TG concentration $>500 \mathrm{mg} / \mathrm{dL}$ or a HDL-C concentration $>100 \mathrm{mg} / \mathrm{dL}$ were excluded [9-11]. Participants with a history of diabetes or a fasting glucose concentration $\geq 126 \mathrm{mg} / \mathrm{dL}$ were also excluded. The remaining 723 individuals (486 women, and 237 men) were included in the present analysis (Fig. 1).

\section{Measurements}

Clinical and biological variables were quantified as previously described [16]. In brief, weight was determined with individuals wearing light clothes and no shoes. Height also was measured without shoes, using a metallic metric tape; waist circumference was measured with a relaxed abdomen using a metallic metric tape on a horizontal plane above the iliac crest; body mass index (BMI) was calculated using the formula weight $(\mathrm{kg}) /$ height $(\mathrm{m})^{2}$. Concentrations of plasma glucose, TG, HDL-C, and fasting plasma insulin were determined after an overnight (12-h) fast. Low-density lipoprotein cholesterol (LDL-C) concentrations were estimated by the Friedewald formula [17]. 


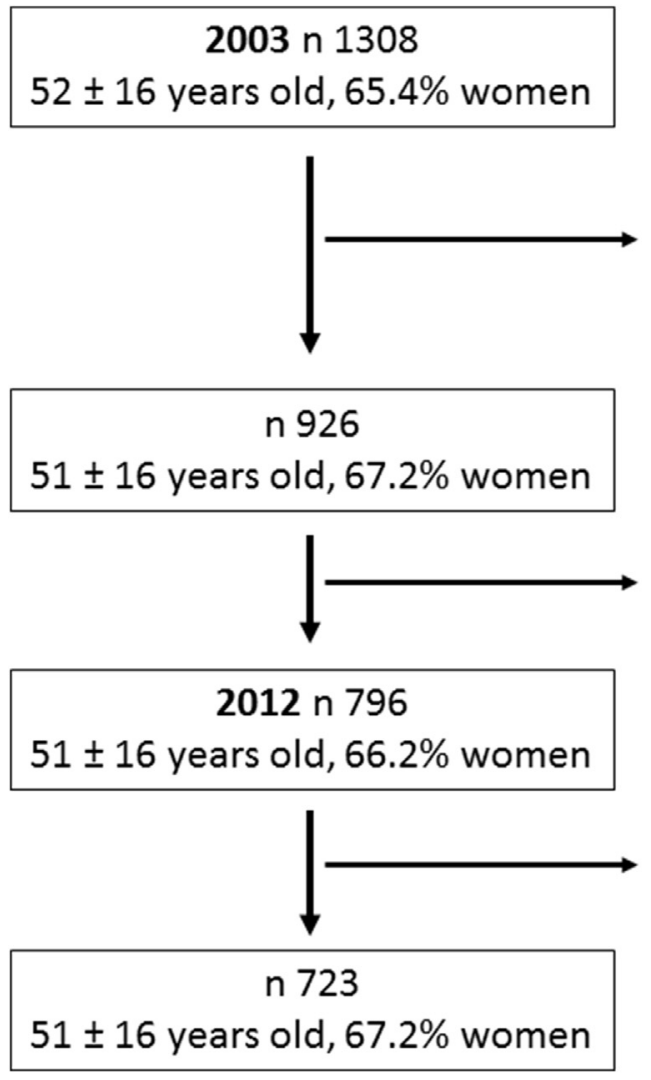

Baseline CVD n 54

$66 \pm 11$ years old, $46.3 \%$ women

Without lipid profile $\mathrm{n} 328$

$49 \pm 17$ years old, $64.0 \%$ women

Without follow-up $\mathrm{n} 130$

$54 \pm 18$ years old, $73.2 \%$ women

TG $>500 \mathrm{mg} / \mathrm{dL}$ or HDL-C $>100$

$\mathrm{mg} / \mathrm{dL}$ or diabetes $\mathrm{n} 73$

$59 \pm 14$ years old, $56,2 \%$ women
Plasma for the insulin measurements was extracted by centrifugation ( $15 \mathrm{~min}$ at 3000 r.p.m.) and frozen at $-20^{\circ} \mathrm{C}$ until assayed. Fasting plasma insulin concentrations were determined using an immunoradiometric assay, with 2 monoclonal antibodies against 2 different epitopes of the insulin molecule. The inter- and intra-assay coefficients of variation were $8.0 \%$ and $3.8 \%$, respectively, with the lowest detectable level of $1.4 \mathrm{pmol} / \mathrm{L}$. The homeostasis model assessment of insulin resistance was calculated with the formula ([Insulin $(\mu \mathrm{U} \mathrm{mL}) \times$ glucose $(\mathrm{mmol} / \mathrm{L}) / 22.5)$. The TG-G index was calculated as the Ln [fasting TG $(\mathrm{mg} /$ $\mathrm{dL}) \times$ fasting glucose $(\mathrm{mg} / \mathrm{dL}) / 2][12]$.

\section{Assessment of endpoints}

The first CVD event, including angina pectoris, myocardial infarction, myocardial revascularization, and fatal or nonfatal stroke was defined as the primary endpoint. Structured interviews were conducted with each participant by specially trained nurses and social workers. The collected data were then evaluated by a highly qualified internist (blinded with respect to the subject's baseline CVD risk factors) to assign a specific outcome for every event. When necessary, available medical records also were reviewed.

\section{Definition of risk groups}

Previously published cut points of TG/HDL-C concentration ratios of 2.5 and $3.5(\mathrm{mg} / \mathrm{dL})$, for women and men, respectively, were used to classify participants as being either at high or low cardiovascular disease risk [9]. These cut points were created because they separated those within the highest quartile of TG/HD-C ratios from the remainder individuals. Consequently, the sample was also divided into quartiles of TG $\times \mathrm{G}$ index, and those individuals in the upper quartile of TG-G index also were classified as being at high risk. Finally, the individuals were also divided into those with the MetS (high risk) or without the MetS (low risk), using the "harmonized" version of the MetS [15], in which three of the following five criteria are required for diagnosis: (1) WC $\geq 102 \mathrm{~cm}$ in men and $\geq 88 \mathrm{~cm}$ in women; (2) HDL-C $<40 \mathrm{mg} / \mathrm{dL}$ in men and $<50 \mathrm{mg} / \mathrm{dL}$ in women; (3) $\mathrm{TG} \geq 150 \mathrm{mg} / \mathrm{dL}$; (4) $\mathrm{SBP} \geq 130 \mathrm{~mm} \mathrm{Hg}$ or DBP $\geq 85 \mathrm{~mm} \mathrm{Hg}$; or (5) fasting plasma glucose $\geq 100 \mathrm{mg} / \mathrm{dL}$.

\section{Statistical analyses}

Continuous baseline variables (age, body mass index, WC, SBP, DBP, glucose, cholesterol, LDL-C, HDL-C, TG, FPI and HOMA-IR) were expressed as mean \pm standard deviation (SD) and compared between risk categories using independent samples ' $t$ ' test. Proportions (sex, current smokers, drugs that patients take) were expressed as percentages and compared by $\chi^{2}$ test.

The relationship between TG/HDL-C ratio concentration ratios and TG $\times$ G index as continuous variables was evaluated using Pearson's $r$ coefficient and the agreement 
between the 2 definitions of high risk with coefficient of concordance (kappa, $k$ ). Receiver operating characteristic (ROC) curves were constructed in order to provide a graphic representation of the relationship between falsepositive (i.e. 1-specificity) and true-positive (sensitivity) detection rates for both, TG $\times$ G and the TG/HDL-C ratio as continuous variables. The area under the ROC curves and $95 \%$ confidence interval $(95 \% \mathrm{CI})$ was used to compare the two indices.

Kaplan-Meier analysis was performed to estimate mean survival time, and the equality of survival distributions for high vs. low risk based on use of the TG/HDL-C, TG $\times$ G, or MetS criteria were tested with log rank (Mantel-Cox). The relative risks for CVD events between individuals above and below the TG/HDL-C sex-specific cut points, in the top quartile of TG $\times \mathrm{G}$ index values vs. the remaining 3 quartiles, and with or without MetS were estimated using three Cox proportional hazard models: (1) unadjusted, (2) adjusted for age and sex, (3) adjusted for sex, age, smoking, LDL-C, BMI, and aspirin, antihypertensive and lipid-lowering drug use. The model-building

Table 1 Demographic and metabolic characteristics of the study population.

\begin{tabular}{llll}
\hline & $\begin{array}{l}\text { Women } \\
(\mathrm{n}=486)\end{array}$ & $\begin{array}{l}\text { Men } \\
(\mathrm{n}=237)\end{array}$ & $\mathrm{p}$ \\
\hline Age (yrs) & $50 \pm 16$ & $51 \pm 16$ & 0.643 \\
Current smokers (\%) & 19.8 & 28.8 & 0.005 \\
$\begin{array}{l}\text { Aspirin use (\%) } \\
\text { Antihypertensive }\end{array}$ & 2.1 & 3.4 & 0.205 \\
$\quad 15.4$ & 15.6 & 0.515 \\
$\quad$ drugs (\%) & & & \\
$\quad$ Lipid-lowering & 3.3 & 3.4 & 0.555 \\
$\quad$ Brugs (\%) & & & \\
WC $\left(\mathrm{kg} / \mathrm{m}^{2}\right)$ & $25.1 \pm 4.5$ & $26.4 \pm 3.7$ & $<0.001$ \\
SBP $(\mathrm{mmHg})$ & $91 \pm 12$ & $96 \pm 12$ & $<0.001$ \\
DBP $(\mathrm{mmHg})$ & $129 \pm 18$ & $135 \pm 18$ & $<0.001$ \\
TG $(\mathrm{mmol} / \mathrm{L})$ & $80 \pm 11$ & $84 \pm 12$ & $<0.001$ \\
Cholesterol (mmol/L) & $1.4 \pm 0.7$ & $1.7 \pm 0.9$ & $<0.001$ \\
LDL-C (mmol/L) & $5.9 \pm 1.3$ & $5.9 \pm 1.2$ & 0.527 \\
HDL-C (mmol/L) & $3.7 \pm 1.0$ & $3.8 \pm 1.0$ & 0.330 \\
FPG (mmol/L) & $1.6 \pm 0.3$ & $1.4 \pm 0.3$ & $<0.001$ \\
\hline
\end{tabular}

Continuous baseline variables were expressed as mean \pm standard deviation and compared by independent samples ' $t$ ' test. Proportions were expressed as percentages and compared by $\chi^{2}$ test. $\mathrm{BMI}=$ body mass index, $\mathrm{WC}=$ waist circumference; $\mathrm{SBP}=$ systolic blood pressure; $\mathrm{DBP}=$ diastolic blood pressure; $\mathrm{TG}=$ triglyceride; LDL-C = low density lipoprotein cholesterol; HDL-C = high-density lipoprotein cholesterol; FPG = fasting plasma glucose; FPI = fasting plasma insulin; HOMA-IR = homeostasis model assessment of insulin resistance. process took place in 3 blocks. In the first block, risk categories were included as categorical variables. In the second block, sex and age were added. Finally, in the third block conditional forward stepwise algorithm methods were used for multivariable analysis and baseline (2003) covariates were added; the probabilities for stepwise were $<0.05$ and $>0.10$ for inclusion and exclusion, respectively. The risk was expressed as hazard ratio (HR) and its 95\% confidence interval. Finally, a further analysis was performed using both risk markers as continuous variables. Since TG $\times$ G index is a log function, the TG/HDL-C ratio was $\log$ transformed and both variables were included as Z-scores.

All significant tests were 2-tailed, and p values $<0.05$ were considered statistically significant. All statistical analyses were performed using SPSS 18.0.

\section{Results}

Demographic, metabolic, and clinical characteristics of the experimental population were previously published [16] and are summarized in Table 1. Men were more often current smokers, but there were no sex differences in usage of aspirin or drugs to lower blood pressure of lipid concentrations. Men were more obese, with higher values for blood pressure and of TG and fasting glucose concentrations.

Values of both risk markers, TG $\times \mathrm{G}$ index and TG/HDL$C$ ratio, were higher in men (Table 2). The cut-point for the upper quartile of $\mathrm{TG} \times \mathrm{G}$ index was 8.98. The correlations between the TG/HDL-C ratio and the TG $\times \mathrm{G}$ index were high in both men $(0.84, p<0.001)$ and women $(0.80$, $\mathrm{p}<0.001$ ), and agreement between the 2 definitions to identify individuals at high risk was highly statistically significant $(\mathrm{k}=0.73, \mathrm{p}<0.001)$. Moreover, as shown in Fig. 2, the area under the ROC curves was almost identical, $0.66(95 \% \mathrm{CI}=0.59-0.74, \mathrm{p}<0.001)$ for TG/HDL-C ratio and $0.67(95 \% \mathrm{CI}=0.60-0.75, \mathrm{p}<0.001)$ for $\mathrm{TG} \times \mathrm{G}$ index.

Table 3 compares the cardio-metabolic risk profile of individuals identified as being at high risk on the basis of each of the 3 indices and it can be seen that somewhat fewer participants were defined as high risk by the TG $\times \mathrm{G}$ index. Since substantial numbers of individuals meet criteria for low or high risk concordantly on the basis of all 3 indices, statistical comparison of individual cardiometabolic risk factors based on the index used for purpose of classification is not appropriate. On the other hand, visual comparison of the data in Table 3 suggests that the

Table 2 Descriptive statistics of Triglyceride plasmatic levels (TG), High-density Lipoprotein Cholesterol plasmatic levels (HDL-C), the Triglyceride-glucose Index $(\mathrm{TG} \times \mathrm{G})$ and the Triglyceride/High-density Lipoprotein Cholesterol Ratio (TG/HDL-C) according sex.

\begin{tabular}{|c|c|c|c|c|c|c|c|c|}
\hline & \multicolumn{4}{|c|}{ Women $(n=486)$} & \multicolumn{4}{|c|}{$\operatorname{Men}(n=237)$} \\
\hline & Mean & SD & Median & Range & Mean & SD & Median & Range \\
\hline TG & 124 & 63 & 106 & $35-398$ & 151 & 80 & 131 & $44-491$ \\
\hline HDL-C & 61 & 12 & 60 & $20-99$ & 54 & 15 & 53 & $27-85$ \\
\hline TG/HDL-C ratio & 2.2 & 1.5 & 1.8 & $0.5-14.6$ & 3.0 & 2.0 & 2.6 & $0.6-13.6$ \\
\hline $\mathrm{TG} \times \mathrm{G}$ index & 8.54 & 0.51 & 8.50 & $7.3-9.9$ & 8.77 & 0.55 & 8.76 & $7.6-10.0$ \\
\hline
\end{tabular}




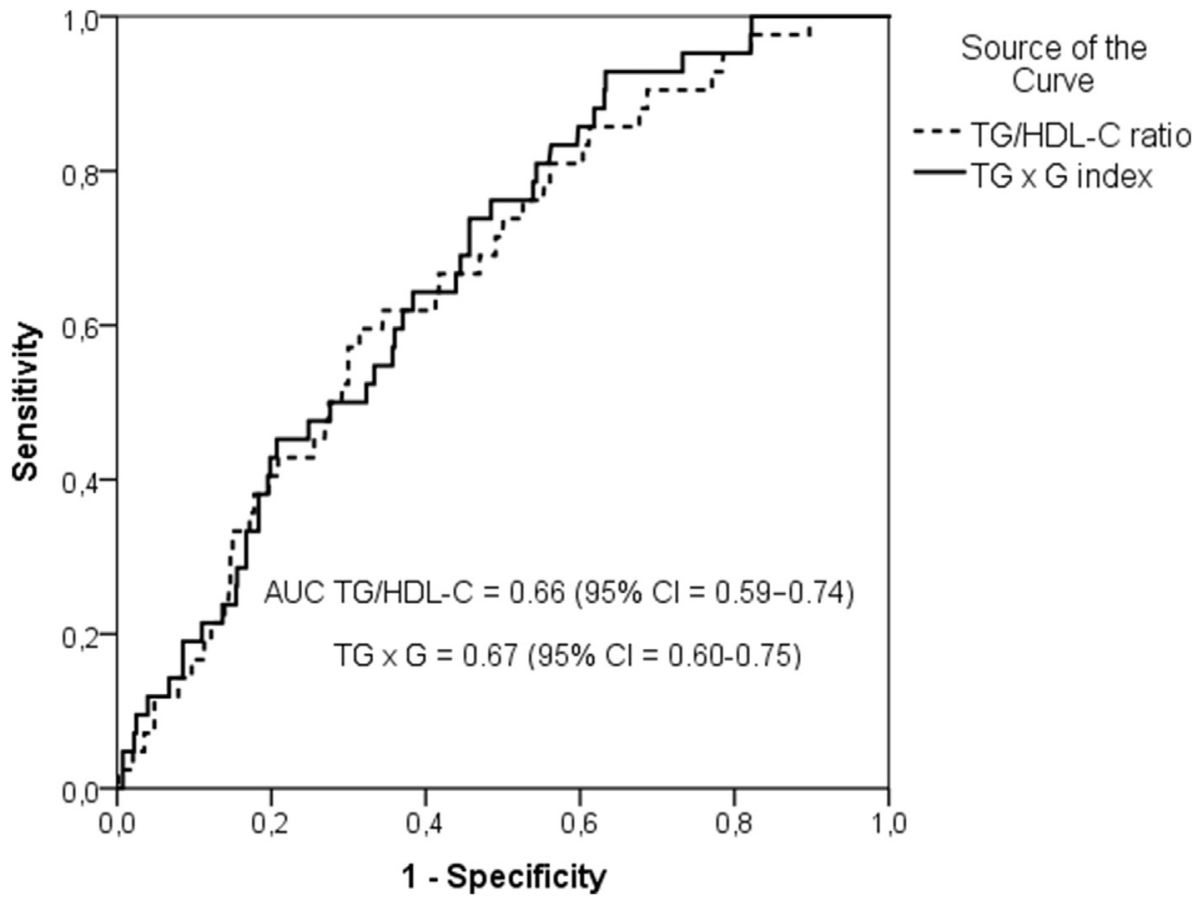

Figure 2 Receiver operating characteristic curves of the triglyceride/high-density lipoprotein cholesterol (TG/HDL-C) ratio and the plasma triglyceride $\times$ glucose $(\mathrm{TG} \times \mathrm{G})$ index for prediction of insulin resistance.

Table 3 Comparison of the cardio-metabolic risk profile in individuals identified as high risk vs. low risk by the Triglyceride-glucose Index $(\mathrm{TG} \times \mathrm{G})$, the Triglyceride/High-density Lipoprotein Cholesterol Ratio (TG/HDL-C) and Metabolic Syndrome Diagnosis (MetS).

\begin{tabular}{|c|c|c|c|c|c|c|c|c|c|}
\hline & \multicolumn{2}{|l|}{$\mathrm{TG} \times \mathrm{G}$ index } & & \multicolumn{2}{|l|}{ TG/HDL-C ratio } & & \multicolumn{2}{|l|}{ MetS } & \\
\hline & $\begin{array}{l}\text { Low } \mathrm{n}=542 \\
(75.0 \%)\end{array}$ & $\begin{array}{l}\text { High } \mathrm{n}=181 \\
(25.0 \%)\end{array}$ & & $\begin{array}{l}\text { Low } \mathrm{n}=517 \\
(71.5 \%)\end{array}$ & $\begin{array}{l}\text { High n }=206 \\
(28,5 \%)\end{array}$ & & $\begin{array}{l}\text { No } \mathrm{n}=497 \\
(68.7 \%)\end{array}$ & $\begin{array}{l}\text { Yes } n=226 \\
(31.3 \%)\end{array}$ & \\
\hline Age (yrs) & $49 \pm 16$ & $54 \pm 14$ & $<0.001$ & $49 \pm 17$ & $53 \pm 14$ & 0.002 & $47 \pm 16$ & $58 \pm 12$ & $<0.001$ \\
\hline Women (\%) & 72.1 & 51.9 & $<0.001$ & 68.3 & 64.6 & 0.191 & 66.6 & 68.6 & 0.609 \\
\hline Current smokers (\%) & 22.7 & 22.8 & 0.991 & 20.2 & 29.3 & 0.008 & 22.4 & 23.6 & 0.727 \\
\hline Aspirin use (\%) & 2.6 & 2.2 & 0.780 & 2.7 & 1.9 & 0.551 & 2.2 & 3.1 & 0.479 \\
\hline Antihypertensive (\%) & 14.2 & 19.3 & 0.099 & 13.0 & 21.8 & 0.003 & 9.7 & 18.3 & $<0.001$ \\
\hline Lipid-lowering (\%) & 3.1 & 3.9 & 0.635 & 3.1 & 3.9 & 0.593 & 2.2 & 5.8 & 0.014 \\
\hline BMI $\left(\mathrm{kg} / \mathrm{m}^{2}\right)$ & $24.8 \pm 4.1$ & $27.8 \pm 3.9$ & $<0.001$ & $24.9 \pm 4.2$ & $27.2 \pm 4.0$ & $<0.001$ & $24.1 \pm 3.7$ & $28.6 \pm 3.9$ & $<0.001$ \\
\hline $\mathrm{WC}(\mathrm{cm})$ & $91 \pm 13$ & $98 \pm 10$ & $<0.001$ & $91 \pm 13$ & $96 \pm 9$ & $<0.001$ & $89 \pm 11$ & $100 \pm 12$ & $<0.001$ \\
\hline SBP (mmHg) & $129 \pm 17$ & $137 \pm 18$ & $<0.001$ & $128 \pm 17$ & $137 \pm 18$ & $<0.001$ & $126 \pm 16$ & $142 \pm 16$ & $<0.001$ \\
\hline DBP (mmHg) & $80 \pm 11$ & $85 \pm 11$ & $<0.001$ & $80 \pm 11$ & $84 \pm 11$ & $<0.001$ & $78 \pm 10$ & $88 \pm 10$ & $<0.001$ \\
\hline TG $(\mathrm{mmol} / \mathrm{L})$ & $1.1 \pm 0.4$ & $2.6 \pm 0.7$ & $<0.001$ & $1.1 \pm 0.4$ & $2.4 \pm 0.8$ & $<0.001$ & $1.2 \pm 0.6$ & $2.1 \pm 0.9$ & $<0.001$ \\
\hline Cholesterol (mmol/L) & $5.7 \pm 1.1$ & $6.6 \pm 1.3$ & $<0.001$ & $5.7 \pm 1.2$ & $6.4 \pm 1.2$ & $<0.001$ & $5.7 \pm 1.2$ & $6.4 \pm 1.2$ & $<0.001$ \\
\hline LDL-C (mmol/L) & $3.6 \pm 1.0$ & $4.1 \pm 1.2$ & $<0.001$ & $3.6 \pm 1.0$ & $4.0 \pm 1.1$ & $<0.001$ & $3.6 \pm 1.0$ & $4.0 \pm 1.1$ & $<0.001$ \\
\hline HDL-C (mmol/L) & $1.6 \pm 0.3$ & $1.4 \pm 0.3$ & $<0.001$ & $1.6 \pm 0.3$ & $1.3 \pm 0.3$ & $<0.001$ & $1.6 \pm 0.3$ & $1.4 \pm 0.3$ & $<0.001$ \\
\hline Glucose $(\mathrm{mmol} / \mathrm{L})$ & $5.1 \pm 0.7$ & $5.7 \pm 0.7$ & $<0.001$ & $5.2 \pm 0.7$ & $5.5 \pm 0.7$ & $<0.001$ & $5.1 \pm 0.6$ & $5.7 \pm 0.6$ & $<0.001$ \\
\hline FPI (Pmol/L) & $48 \pm 23$ & $65 \pm 46$ & $<0.001$ & $47 \pm 23$ & $64 \pm 44$ & $<0.001$ & $46 \pm 23$ & $64 \pm 41$ & $<0.001$ \\
\hline HOMA-IR & $1.5 \pm 1.0$ & $2.2 \pm 1.7$ & $<0.001$ & $1.5 \pm 1.0$ & $2.1 \pm 1.6$ & $<0.001$ & $1.5 \pm 1.0$ & $2.1 \pm 1.5$ & $<0.001$ \\
\hline
\end{tabular}

Continuous baseline variables were expressed as mean \pm standard deviation and compared by independent samples ' $t$ ' test. Proportions were expressed as percentages and compared by $\chi^{2}$ test. BMI $=$ body mass index, WC $=$ waist circumference; SBP $=$ systolic blood pressure; $\mathrm{DBP}=$ diastolic blood pressure; TG = triglyceride; LDL-C = low density lipoprotein cholesterol; HDL-C = high-density lipoprotein cholesterol; $\mathrm{FPG}=$ fasting plasma glucose; FPI = fasting plasma insulin; HOMA-IR = homeostasis model assessment of insulin resistance.

cardio-metabolic profiles of the three different groups were relatively comparable, with relatively minor differences related to the components of the index used. It is noteworthy that TG/HDL-C ratio and MetS identified as high risk a similar proportion of men and women but the TG $\times$ G index identified significantly more men $(\mathrm{p}<0.001)$.
In the follow-up period (5781 subjects-year, mean $8.16 \pm 1.3$ years), there were 42 CVD events: 24 nonfatal coronary events (14 angina pectoris, 6 myocardial infarction, and 4 myocardial revascularization), 8 fatal coronary events (4 myocardial infarction, 2 unspecific coronary heart disease, and 2 sudden death), 7 nonfatal strokes, and 3 fatal strokes. There also were 30 non-cardiovascular 
deaths. CVD events were documented in approximately more than twice as many subjects classified at high risk by both, baseline TG/HDL-C ratios (12.2 vs. $5.3 \%$ subjects/10 years, $\mathrm{p}=0.005)$ or TG $\times \mathrm{G}$ index (13.4 vs. $5.3 \%$ subjects/ 10 years, $\mathrm{p}=0.002$ ). These results resembled those using MetS criteria (13.4\% vs. $4.5 \%$ of subjects/10 years, $\mathrm{p}<0.001$ ).

Table 4 shows the HR of developing CVD. These results show that both unadjusted and adjusted for age and sex ratios were significantly increased in individuals at high risk as defined by their TG $\times \mathrm{G}$ Index, TG/HDL-C ratio, or MetS diagnosis. Furthermore, age and sex adjusted HR were comparable when individuals were identified with all three of the indices.

Furthermore, when analyzed as continuous variables both, TG $\times$ G index and TG/HDL-C ratio predicted a similar augmentation of risk (Table 5). However, after adjustment for BMI, LDL-C, smoking and use of aspirin, antihypertensive and lipid-lowering drugs, persons classified on the basis of the TG/HDL-C ratio or the MetS maintain their statistical significance HRs, but the HR of CVD was only of a marginal statistical significance $(\mathrm{p}=0.087)$ in persons identified the TG $\times$ G index (Table 4 )

Figure 3 shows free events cumulative survival, adjusted by sex, age and covariates, in individuals classified as high versus low risk using the TG/HDL-C ratio (a), the upper quartile of TG $\times \mathrm{G}$ index (b) and MetS diagnostic criteria (c).

\section{Discussion}

Despite the similarities between the three approaches to identify insulin resistance and enhanced cardio-metabolic risk, the $\mathrm{TG} \times \mathrm{G}$ index seems to be somewhat less successful than either the TG/HDL-C ratio or the MetS in predicting incident CVD. The relative similarity in the increased risk when both indices are analyzed as continuous variables does not diminish the need to have specific cutoff values to use in clinical for application in clinical practice. When adjusted for relevant covariates, e.g., age, sex, BMI. LDL-cholesterol, smoking, and use of aspirin and blood pressure or lipid lowering drugs (Table 4), the hazard ratio for incident CVD remained statistically significant when evaluated by the TG/HDL-C ratio $(p=0.021)$ or the MetS criteria $p=0.036)$, but not by the $T G \times G$ index $(\mathrm{p}=0.087)$.

In view of the many similarities that have been shown to exist between the TG $\times$ G index and the TG/HDL-C ratio, it was surprising that, after the adjustment for covariates, the TG $\times G$ index was less successful in identifying incident CVD in individuals without diabetes. We can only speculate as why the current analysis found the $T G \times G$ index to be a less robust predictor of incident CVD than described by Sánchez-Íñigo et al. [14]. The experimental population in their study seems similar to the current one in terms age, race/ethnicity, and duration of follow-up. Furthermore, using the same cut-point (8.43) to define the upper quartile of the TG $\times \mathrm{G}$ index as proposed by Sánchez-Íñigo et al. [14] did not substantially change the hazard ratio. However, there are two important differences between the studies. Firstly, Sánchez-Íñigo et al. [14] included patients with diabetes, whereas they were excluded from this analysis. Inclusion of subjects with diabetes would have two effects; 1 ) increasing the TG $\times \mathrm{G}$ index to a degree not attainable when patients with diabetes are excluded from the population; and 2) increasing CVD as a consequence of the multiple risk factors present in patients with manifest diabetes. These two effects,

Table 4 Hazard Ratios (HR) of cardiovascular events occurring in individuals defined as high risk using Triglyceride-glucose Index (TG $\times$ G), the Plasma Triglyceride/High-density Lipoprotein Cholesterol Ratio (TG/HDL-C) and the Metabolic Syndrome Diagnosis (MetS).

\begin{tabular}{|c|c|c|c|c|c|c|c|c|c|c|}
\hline & \multirow{3}{*}{$\begin{array}{l}\text { Crude incidence } \\
100 \text { subjects/10 years }\end{array}$} & \multicolumn{9}{|c|}{ Cox models } \\
\hline & & \multicolumn{3}{|c|}{ Unadjusted } & \multicolumn{3}{|c|}{ Adjusted for sex and age } & \multicolumn{3}{|c|}{ Adjusted for sex, age, and covariates } \\
\hline & & HR & $95 \% \mathrm{CI}$ & $\mathrm{p}$ & $\mathrm{HR}$ & $95 \% \mathrm{CI}$ & $\mathrm{p}$ & $\mathrm{HR}$ & $95 \% \mathrm{CI}$ & $\mathrm{p}$ \\
\hline TG/HDL-C ratio & $\begin{array}{l}\text { low } 5.3(\mathrm{n}=517) \\
\text { high } 12.2(\mathrm{n}=206)\end{array}$ & 2.34 & $1.28-4.29$ & 0.006 & 2.19 & $1.19-4.02$ & 0.012 & 2.06 & $1.12-3.80$ & 0.021 \\
\hline $\mathrm{TG} \times \mathrm{G}$ Index & $\begin{array}{l}\text { low } 5.3(\mathrm{n}=542) \\
\text { high } 13.4(\mathrm{n}=181)\end{array}$ & 2.58 & $1.40-4.73$ & 0.002 & 1.92 & $1.04-3.59$ & 0.036 & 1.72 & $0.93-3.19$ & 0.087 \\
\hline MetS & $\begin{array}{l}\text { no } 4.5(\mathrm{n}=497) \\
\text { yes } 13.4(\mathrm{n}=226)\end{array}$ & 3.02 & $1.64-5.54$ & $<0.001$ & 1.93 & $1.04-3.58$ & 0.036 & 1.93 & $1.04-3.58$ & 0.036 \\
\hline
\end{tabular}

a BMI, LDL-cholesterol, smoking, aspirin, antihypertensive and lipid-lowering drug use.

Table 5 Hazard Ratios (HR) of cardiovascular events occurring according Triglyceride-glucose Index (TG $\times$ G) and Triglyceride/High-density Lipoprotein Cholesterol Ratio (TG/HDL-C) analyzed as continuous variables.

\begin{tabular}{|c|c|c|c|c|c|c|c|c|c|}
\hline & \multicolumn{9}{|c|}{ Cox Models } \\
\hline & \multicolumn{3}{|c|}{ Unadjusted } & \multicolumn{3}{|c|}{ Adjusted for sex and age } & \multicolumn{3}{|c|}{ Adjusted for sex, age, and covariates ${ }^{a}$} \\
\hline & HR & $95 \% \mathrm{CI}$ & $\mathrm{p}$ & HR & $95 \% \mathrm{CI}$ & $\mathrm{p}$ & $\overline{\mathrm{HR}}$ & $95 \% \mathrm{CI}$ & $\mathrm{p}$ \\
\hline TG/HDL-C ratio & 1.64 & $1.24-2.17$ & $<0.001$ & 1.55 & $1.15-2.09$ & 0.004 & 1.53 & $1.11-2.11$ & 0.010 \\
\hline $\mathrm{TG} \times \mathrm{G}$ index & 1.76 & $1.31-2.35$ & $<0.001$ & 1.54 & $1.12-2.12$ & 0.009 & 1.46 & $1.03-2.08$ & 0.033 \\
\hline
\end{tabular}

a BMI, LDL-cholesterol, smoking, aspirin, antihypertensive and lipid-lowering drug use. 

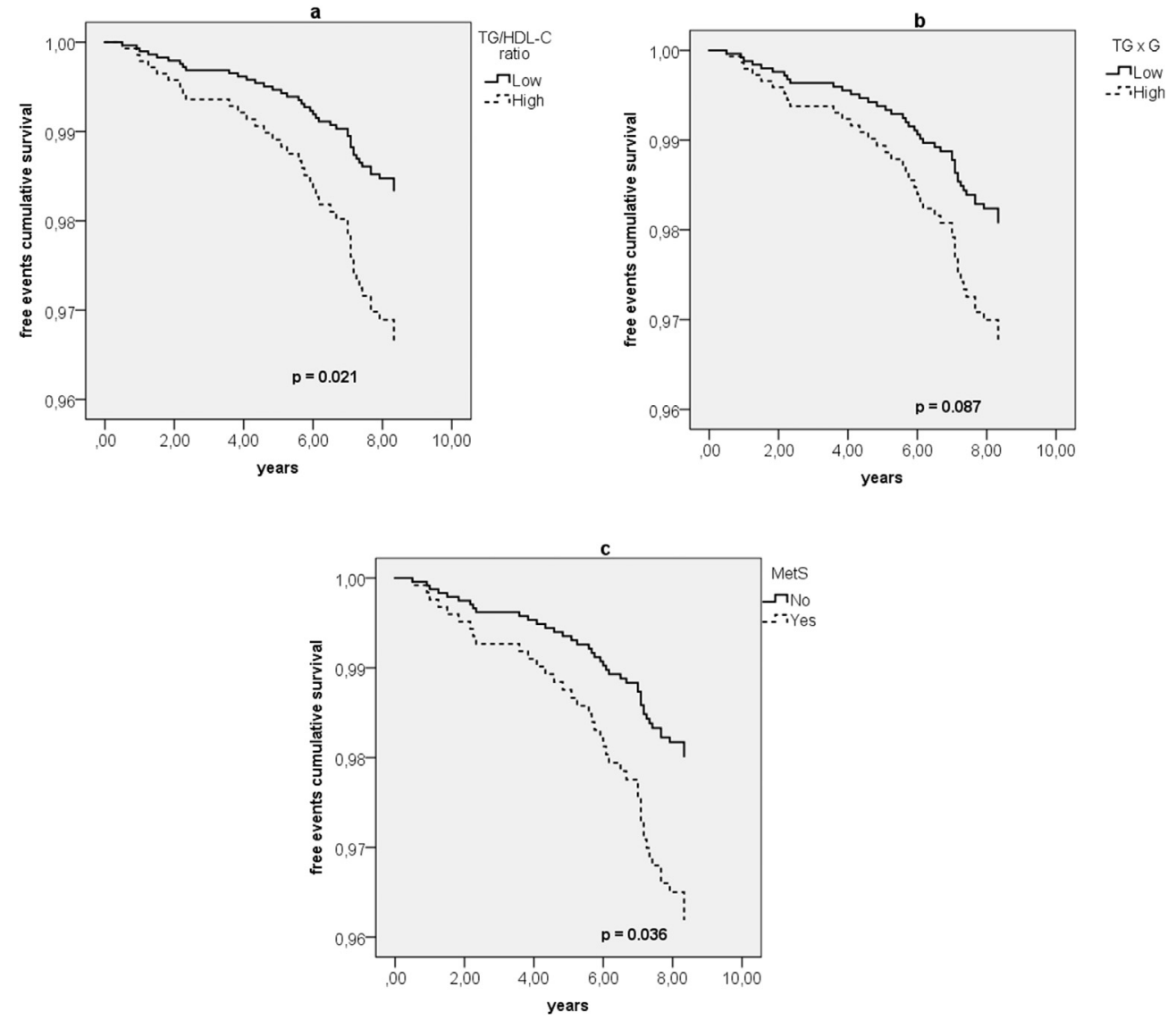

Figure 3 Free events cumulative survival, adjusted by sex, age, LDL-C levels, BMI, smoking, and use of antihypertensive, lipid-lowering drugs and aspirin, in individuals classified as high versus low risk using the plasma triglyceride/high density-lipoprotein cholesterol ratio (TG/HDL-C) (a), the plasma triglyceride $\times$ glucose index $(\mathrm{TG} \times \mathrm{G})(\mathrm{b})$ and metabolic syndrome (MetS) diagnostic criteria (c).

acting in concert, could help explain why SánchezÍñigo et al. [14] were able to show that the TG $\times$ G index predicted incident CVD when patients with diabetes were included in the experimental population, whereas their absence may account for the failure to discern a comparable degree of predictability in the current study. Furthermore, the observations by Steele et al. [18]., Faerch et al. [19] and the data of the Emerging Risk Factors Collaboration [20] suggest that, in the absence of diabetes, mild elevation of fasting plasma glucose, per se, has relatively little adverse effect on macrovascular disease. Supporting this notion, our working group, in a recently published study, shows that the risk to develop CVD events is accentuated in individuals with prediabetes mellitus who are also insulin resistant [21].

Secondly, the same proportion of women and men were identified as high risk using the TG/HDL-C ratio and MetS diagnosis, whereas the TG $\times \mathrm{G}$ index identified more men than women. An increase in proportion of men at high risk for CVD was also true of the report by Sánchez-Íñigo et al. [14], suggesting the potential importance of sex differences, and the need to take sex-specific effects into account as is done when using the TG/HDL-C ratio or the MetS diagnostic criteria.

Although the analysis and the results appear straightforward, there are some major concerns that must be emphasized. In the first place, the CVD events were recorded based in a structured interview. Subsequently, the collected data were evaluated by a qualified internist (blinded with respect to the subject's baseline CVD risk factors) to assign a specific outcome for every event. Also, when necessary, available medical records were reviewed. Secondly, since the population was quite homogeneous, the findings might not apply to ethnic/racial groups that are not primarily of European descent. Finally, there were a relatively small number of documented CVD events and the adjusted HR of incident CVD using the TG $\times$ G index was of borderline significance, it is possible that a larger sample size could lead to a different result. On the other hand, the number of CVD events was sufficient to document statistically significant relationships between high CVD risk and increased CVD events when persons were classified by either the TG/HDL-C ratio of the MetS criteria.

Although far from definitive, our results suggest that the apparent similarity of the TG $\times$ G index and the TG/ HDL-C ratio to identify apparently healthy persons who are insulin resistant [12,13] does not mean that the 2 indices have the same ability to predict CVD events in $\mathrm{p}$. Thus, despite the limitations of the current analysis outlined above, the fact that increased CVD was seen in high risk persons identified by either the TG/HDL-C ratio or the MetS diagnostic criteria raises questions as to the ability of 
the TG $\times$ G index to accomplish the same task. The ultimate decision as to the clinical utility of the TG $\times$ G index will depend upon results of similar analyses by investigators with larger data bases.

\section{Acknowledgements}

This study could not have been conducted without the help of the nurses from the "Hospital Municipal of Rauch".

\section{References}

[1] Yeni-Komshian H, Carantoni M, Abbasi F, Reaven GM. Relationship between several surrogate estimates of insulin resistance and quantification of insulin-mediated glucose disposal in 490 healthy, nondiabetic volunteers. Diabetes Care 2000;23:171-5.

[2] Zavaroni I, Bonini L, Gasparini P, Barilli AL, Zuccarelli A, Dall'Aglio E, et al. Hyperinsulinemia in a normal population as a predictor of non-insulin-dependent diabetes mellitus, hypertension, and coronary heart disease: the Barilla factory revisited. Metabolism 1999;48:989-94.

[3] Yip J, Facchini FS, Reaven GM. Resistance to insulin-mediated glucose disposal as a predictor of cardiovascular disease. J Clin Endocrinol Metab 1998;83:2773-6.

[4] Facchini FS, Hua N, Abbas F, Reaven GM. Insulin resistance as a predictor of age-related diseases. J Clin Endocrinol Metab 2001; 86:3574-8.

[5] Reaven G. Insulin resistance and coronary heart disease in nondiabetic individuals. Arterioscler Thromb Vasc Biol 2012;32: 1754-9.

[6] McLaughlin T, Reaven G, Abbasi F, Lamendola C, Saad M, Waters D, et al. Is there a simple way to identify insulin-resistant individuals at increased risk of cardiovascular disease? Am J Cardiol 2005;96: 399-404.

[7] Reaven GM. Wanted! A standardized measurement of plasma insulin concentration. Arterioscler Thromb Vasc Biol 2011;31: 954-5.

[8] Laws A, Reaven GM. Evidence for an independent relationship between insulin resistance and fasting plasma HDL-cholesterol, triglyceride and insulin concentrations. J Int Med 1992;231: 25-30.

[9] Salazar MR, Carbajal HA, Espeche WG, Leiva Sisnieguez CE, Balbín E, Dulbecco CA, et al. Relation among the plasma triglyceride/high-density lipoprotein cholesterol concentration ratio, insulin resistance and associated cardio-metabolic risk factors in men and women. Am J Cardiol 2012;109:1749-53.

[10] Salazar MR, Carbajal HA, Espeche WG, Leiva Sisnieguez CE, March CE, Balbín E, et al. Comparison of the abilities of the plasma triglyceride/high density lipoprotein cholesterol ratio and the metabolic syndrome to identify insulin resistance. Diab Vasc Dis Res 2013;10:346-52.

[11] Salazar MR, Carbajal HA, Espeche WG, Aizpurúa M, Leiva Sisnieguez CE, March CE, et al. Cardiovascular disease risk and outcome: use of the plasma triglyceride/high-density lipoprotein cholesterol concentration ratio versus metabolic syndrome criteria. J Intern Med 2013;273:595-601.

[12] Guerrero-Romero F, Simental-Mendía LE, González-Ortiz M, Martínez-Abundis E, Ramos-Zavala MG, Hernández-González SO, et al. The product of triglycerides and glucose, a simple measure of insulin sensitivity. Comparison with the euglycemichyperinsulinemic clamp. J Clin Endocrinol Metab 2010;95: 3347-51.

[13] Abbasi F, Reaven GM. Comparison of two methods using plasma triglyceride concentration as a surrogate estimate of insulin action in nondiabetic subjects: triglycerides $\times$ glucose versus triglyceride/high-density lipoprotein cholesterol. Metabolism 2011;60:1673-6.

[14] Sánchez-Íñigo L, Navarro-González D, Fernández-Montero A Pastrana-Delgado J, Martínez JA. The TyG index may predict the development of cardiovascular events. Eur J Clin Invest 2016; 46(2):189-97.

[15] Alberti KG, Eckel RH, Grundy SM, Zimmet PZ, Cleeman JI, Donato KA, et al. Harmonizing the metabolic syndrome: a joint interim statement of the International Diabetes Federation task force on epidemiology and prevention; National Heart, Lung, and Blood Institute; American Heart Association; World Heart Federation; International Atherosclerosis Society; International Association for the Study of Obesity. Circulation 2009;120:1640-5.

[16] Salazar MR, Carbajal HA, Aizpurúa M, Riondet B, Rodrigo HF Rechifort $\mathrm{V}$, et al. Decrease of blood pressure by community-based strategies. Medicina (B Aires) 2005;65:507-12.

[17] Friedewald WT, Levy RI, Fredrickson DS. Estimation of the concentration of low-density lipoprotein cholesterol in plasma, without use of the preparative ultracentrifuge. Clin Chem 1972;18: 499-502.

[18] Steele AM, Shields BM, Wensley KJ, Colclough K, Ellard S, Hattersley AT. Prevalence of vascular complications among patients with glucokinase mutations and prolonged mild hyperglycemia. JAMA 2014;311:279-86.

[19] Faerch K, Bergman B, Perreault L. Does insulin resistance drive the association between hyperglycemia and cardiovascular risk? PLoS One 2012; 7:e39260.

[20] Emerging Risk Factors Collaboration, Sarwar N, Gao P, Seshasai SR, Gobin R, Kaptoge S, Di Angelantonio E, et al. Diabetes mellitus, fasting blood glucose concentration, and risk of vascular disease: a collaborative meta-analysis of 102 prospective studies. Lancet 2010;375:2215-22.

[21] Salazar MR, Carbajal HA, Espeche WG, Aizpurúa M, Leiva Sisnieguez CE, Leiva Sisnieguez BC, et al. Insulin resistance: the linchpin between prediabetes and cardiovascular disease. Diab Vasc Dis Res 2016:13:157-63. 